Uijen, A.A., Schers, H.J., Schene, A.H., Schellevis, F.G., Lucassen, P., Bosch, W.J.H.M. van depn Experienced continuity of care in patients at risk for depression in primary care. European

\begin{tabular}{|l|l|}
$\begin{array}{l}\text { Postprint } \\
\text { Version }\end{array}$ & 1.0 \\
\hline Journal website & http://informahealthcare.com/doi/abs/10.3109/13814788.2013.828201 \\
\hline Pubmed link & $\underline{\text { http://www.ncbi.nlm.nih.gov/pubmed/24033228 }}$ \\
\hline DOI & $10.3109 / 13814788.2013 .828201$ \\
\hline
\end{tabular}

This is a NIVEL certified Post Print, more info at http://www.nivel.eu

\title{
Experienced continuity of care in patients at risk for depression in primary care
}

\author{
AnNemarie A. Uijen, Henk J. SCHERs, Aart H. SChene, Francois G. SCHElleVis, \\ Peter LuCASSEN, AND WIL J. H. M. VAN DEN BOSCH
}

\footnotetext{
${ }^{1}$ Department of Primary and Community Care 117, Radboud University Nijmegen Medical Centre, Nijmegen, The Netherlands

${ }^{2}$ Department of Psychiatry, Academic Medical Center, University of Amsterdam, Amsterdam, The Netherlands

${ }^{3}$ Netherlands Institute for Health Services Research (NIVEL), Utrecht, The Netherlands

${ }^{4}$ Department of General Practice, EMGO Institute for Health and Care Research, VU University Medical Centre, Amsterdam, The Netherlands
}

\begin{abstract}
Background: Existing studies about continuity of care focus on patients with a severe mental illness.

Objectives: Explore the level of experienced continuity of care of patients at risk for depression in primary care, and compare these to those of patients with heart failure.

Methods: Explorative study comparing patients at risk for depression with chronic heart failure patients. Continuity of care was measured using a patient questionnaire and defined as (1) number of care providers contacted (personal continuity); (2) collaboration between care providers in general practice (team continuity) (six items, score 1-5); and (3) collaboration between GPs and care providers outside general practice (cross-boundary continuity) (four items, score $1-5)$.

Results: Most patients at risk for depression contacted several care providers throughout the care spectrum in the past year. They experienced high team continuity and low cross-boundary continuity. In their general practice, they contacted more different care providers for their illness than heart failure patients did $(P<0.01)$. Patients at risk for depression experienced a slightly better collaboration between these care providers in their practice: a mean score of 4.3 per item compared to 4.0 for heart failure patients $(P=0.03)$. The perceived cross-boundary continuity, however, was reversed: a mean score of 3.5 per item for patients at risk for depression, compared to 4.0 for heart failure patients $(P=0.01)$.
\end{abstract}


Uijen, A.A., Schers, H.J., Schene, A.H., Schellevis, F.G., Lucassen, P., Bosch, W.J.H.M. van depn Experienced continuity of care in patients at risk for depression in primary care. European Journal of General Practice: 2014, 20(3), 161-166

Conclusion: The explorative comparison between patients at risk for depression and heart failure patients shows small differences in experienced continuity of care. This should be analysed further in a more robust study.

\section{INTRODUCTION}

Continuity of care is a core value of general practice. Research has shown that patients value continuity (1). Patients with chronic somatic illnesses, such as chronic obstructive pulmonary disease (COPD) and heart failure, are known to experience high levels of continuity, both personal continuity (contacting the same care provider) and continuity defined as communication and cooperation between care providers in and outside general practice $(2,3)$. Less is known for patients in primary care suffering from chronic mental health conditions. To our knowledge, there is no study that analyses the level of experienced continuity of care in primary care patients with a chronic mental health problem.

Existing studies have focused solely on patients with a severe mental illness (e.g. schizophrenia or bipolar disorder) recruited in secondary care. These studies show that seeing the same care provider facilitates recovery from a chronic severe mental illness and is related to better quality of life (4). Continuity of care-not only contacting the same care provider but also good communication and cooperation between care providers - is associated with better quality of life, better community functioning, lower severity of symptoms, greater service satisfaction and lower health care costs in patients with a severe chronic mental illness $(5,6)$. At the same time that we wanted to study continuity of care in primary care patients with a chronic mental health condition, another research project started to screen for depression in a primary care population (7). We were able to join this project. The aim of the study presented here was, therefore, to explore the level of experienced continuity of care in patients at risk for depression recruited in primary care. Moreover, since we used the same questionnaire as had been used in a previous study on continuity of care in patients with COPD and heart failure $(2,3)$, we had the opportunity to compare the level of experienced continuity of care of patients at risk for depression with those of patients with a chronic somatic illness recruited in primary care. As we did not have all data on the experienced continuity of COPD patients, we decided to compare patients at risk for depression with heart failure patients. We test the hypothesis that patients at risk for depression experience at least the same level of continuity of care as patients with heart failure.

\section{METHODS}

\section{Design}

In the Netherlands, every patient is enlisted with a general practitioner (GP) who is a gatekeeper for specialist care. Most heart failure patients in the Netherlands obtain non-structured medical care for their heart failure by their GP. Nurse practitioners are more and more taking over the monitoring of blood pressure and other risk factors. In comparison, most patients at risk for depression also obtain care from their GP. Mainly, both groups will take the initiative to visit their GP themselves. 
Uijen, A.A., Schers, H.J., Schene, A.H., Schellevis, F.G., Lucassen, P., Bosch, W.J.H.M. van depn Experienced continuity of care in patients at risk for depression in primary care. European Journal of General Practice: 2014, 20(3), 161-166

The level of continuity of care was studied in patients at risk for depression using a cross-sectional analysis. This study was part of a project that was designed to screen for depression in a primary care population $(7,8)$. A secondary data analysis was performed for the current explorative comparison with heart failure patients (2). Data were combined from the screening for depression study $(7,8)$ with data from a study on heart failure in primary care (2).

\section{Setting and participants}

Patients at risk for depression. From 2006-2007, patients between 18 and 70 years of age who were at high risk for depression (9-11) were included in 23 general practices (30 GPs, seven nurse practitioners specialized in mental health care) in two regions in the Netherlands: patients with medically unexplained symptoms according to their GP for at least three months (GPs selected these patients from consultation lists within four weeks), frequent attenders (10\% most frequently consulting men and women in two age groups (18-44 and 45-70 years)) and patients with a newly presented mental health problem up to three months prior to the selection date (diagnosis in the chapters $\mathrm{P}$ or $\mathrm{Z}$ in the ICPC (International Classification of Primary Care) in the electronic patient database; besides, we also searched with the predefined free text words: anxiety; worrying; sadness; stress; feeling down; and insomnia). GPs received a list of individuals selected by the research team. They excluded those people who were already recognized by the GP as suffering from schizophrenia; psychosis; bipolar disorder; major depressive disorder; serious somatic disease; mental retardation; or having difficulties with Dutch or English language. All remaining patients were invited to fill out a questionnaire on how they experienced continuity of care (see below). More information regarding the screening study has been previously published (7).

Patients with heart failure. Experienced continuity of care of chronic heart failure patients was measured by using baseline data from another cross-sectional study, in which we used other practices and other exclusion criteria than described above (2). The heart failure patients had been recruited in 42 Dutch primary care practices (72 GPs, 38 nurse practitioners specialized in cardiovascular care) in the period 20052006. We asked the GP to assess whether the patients that the researchers selected from the electronic medical record as having a diagnosis of chronic heart failure (ICPC K77), met the diagnostic criteria of heart failure. All patients with a diagnosis of chronic heart failure according to the GP were eligible to be included. Patients were excluded if they had a terminal illness, a cognitive impairment, Dutch language problems or when the GP decided that patients should not be involved in the study for other reasons. All remaining patients were invited to fill out a questionnaire on how they experienced continuity of care (see below). More information regarding this study has been specified previously (2).

\section{Measurement instrument: experienced continuity of care}

Patients' experienced continuity of care was measured using a questionnaire including 12 items. The questionnaire was sent by mail. In case of non- response, a reminder was sent after two weeks.

The questionnaire was based on 30 patient interviews that were conducted as part of a study on continuity of care (12). Principal component analysis in a population of 
Uijen, A.A., Schers, H.J., Schene, A.H., Schellevis, F.G., Lucassen, P., Bosch, W.J.H.M. van depn Experienced continuity of care in patients at risk for depression in primary care. European Journal of General Practice: 2014, 20(3), 161-166

chronically ill patients from general practice (both patients with a mental and somatic illness) revealed three dimensions of continuity in the questionnaire, corresponding to the dimensions captured in the literature $(13,14)$ :

- Personal continuity (two items): number of care providers that patients contacted in general practice (GPs, nurse practitioners) and overall (both inside and outside general practice) for their illness in the past year; this last item was missing in the study on heart failure patients.

- Team continuity in general practice: the extent to which care providers in general practice (GPs, nurse practitioners) have knowledge of the patient and communicate and cooperate with each other (six items); items were scored on a five-point scale ( 1 = never, 5 = always).

- Cross-boundary continuity: the extent to which GPs and care providers outside general practice communicate and cooperate with each other (four items); items were scored on a five-point scale ( 1 = never, 5 = always).

\section{Analysis}

\section{[TABLE 1]}

The results on the items measuring personal, team and cross-boundary continuity were explored, and the total scores of team continuity (ranging from 6 to 30) and cross-boundary continuity (ranging from 4 to 20) were calculated for both groups. The answers on the negatively keyed question were recorded.

Cases were excluded when half or more of the items of one of the continuity dimensions were missing, i.e. three or more questions on team continuity or two or more questions on cross-boundary continuity. All remaining missing values were imputed by the patient's mean of the non-missing items (15).

Chi-square testing was used to compare the personal continuity score between the two patient groups. A $P$-value $<0.05$ was considered statistically significant.

A multivariable analysis using a general linear model was conducted to compare the total scores of team and cross-boundary continuity between the two study groups. In this model, team and cross-boundary continuity were included as outcomes and age and sex were controlled for. As no dependency of the outcomes within practices existed when controlling for the two patient groups, a multilevel analysis was not deemed necessary.

\section{RESULTS}

\section{[TABLE 2]}

The continuity questionnaire was sent to 366 patients at risk for depression and 264 (72\%) were returned and analysed. The questionnaire was also sent to 461 heart failure patients, and finally 327 (71\%) were analysed. Table 1 shows the age and sex distributions of both study groups. 
Uijen, A.A., Schers, H.J., Schene, A.H., Schellevis, F.G., Lucassen, P., Bosch, W.J.H.M. van depn Experienced continuity of care in patients at risk for depression in primary care. European Journal of General Practice: 2014, 20(3), 161-166

\section{Experienced continuity in patients at risk for depression}

Table 2 shows that almost half of patients at risk for depression (45\%) answered to have contacted one care provider in general practice and 53\% contacted two or more care providers across care settings during the past year.

Most patients experienced a high level of communication and cooperation between care providers in general practice. In total, $15 \%$ of patients experienced maximum team continuity in general practice (score 30 ), while only 3\% of patients experienced a very low level of team continuity (total score 12 or less). In comparison, $5 \%$ of patients experienced maximum cross-boundary continuity (score 20), and 23\% of patients experienced a very low level of cross-boundary continuity (total score eight or less).

\section{Comparison with heart failure patients}

\section{[TABLE 3]}

Heart failure patients contacted less care providers in general practice for their illness in the past year $(P<0.01)$ (Table 2$)$. In total, 38\% of heart failure patients experienced maximum team continuity in general practice (score 30 ), while only $8 \%$ of patients experienced a very low level of team continuity (total score 12 or less). Besides, $51 \%$ of patients experienced maximum cross-boundary continuity (score 20 ), and $9 \%$ of patients experienced a very low level of cross-boundary continuity (total score eight or less).

Table 3 shows the estimated marginal means (controlled for age and sex) of the team and cross-boundary continuity scores in both patient groups. Patients at risk for depression experienced better collaboration between care providers in general practice than heart failure patients, although the differences are small: the mean overall scores of team continuity was 25.7 and 23.7, respectively $(P=0.03)$, corresponding with average item scores of 4.3 ('often') and 4.0 ('often'), respectively. Patients at risk for depression experienced less collaboration between GP and care providers outside general practice than heart failure patients: the mean overall scores of cross-boundary continuity was 14.0 and 16.1, respectively ( $P=$ 0.01 ), corresponding with average item scores of 3.5 ('sometimes/often') and 4.0 ('often'), respectively.

\section{DISCUSSION}

\section{Main findings}

We found that $45 \%$ of patients at risk for depression contacted one care provider in general practice, and 53\% contacted two or more care providers across care settings. Most patients experienced a high level of collaboration between care providers in general practice, but $23 \%$ of patients experienced a low level of collaboration between care settings.

In comparison to heart failure patients, patients at risk for depression contacted more care providers in general practice (less personal continuity). However, they experienced a slightly higher team continuity. Patients at risk for depression experienced somewhat less collaboration between GP and care providers outside general practice (less cross-boundary continuity). However, the differences between both study groups were small, and as the minimal clinically important change in the 
Uijen, A.A., Schers, H.J., Schene, A.H., Schellevis, F.G., Lucassen, P., Bosch, W.J.H.M. van depn Experienced continuity of care in patients at risk for depression in primary care. European Journal of General Practice: 2014, 20(3), 161-166

continuity score has not yet been determined, the clinical meaning of these differences is unclear.

\section{Strengths and limitations}

To our knowledge, this is the first study exploring the level of experienced continuity of care in primary care patients at risk for depression.

The comparison between patients at risk for depression and patients with heart failure has some limitations. First, patients with heart failure can also be at risk for a depression and vice versa, so both groups might not be completely distinct. Second, possible differences in co-morbidity might affect the experienced continuity of care. Third, the age and sex differences between both groups make a comparison more difficult, although we controlled for these variables. Last, the recruitment of the different patient groups differed because we first measured continuity of care in both groups independently and then noticed great differences, which we wanted to describe. Patients at risk for depression should have contacted general practice at least once in the past year according to the inclusion criteria while heart failure patients were identified based on a diagnosis in the medical record regardless of the number of contacts with general practice. This makes it harder to compare scores of personal continuity.

Another limitation is the possible recall bias. In total, 20\% of patients at risk for depression answered to have contacted no care provider in general practice in the past year. However, according to the inclusion criteria, all patients should have contacted general practice at least once in the past year. Depending on the symptoms of the patient consulting the GP, patients were asked about the continuity of care for their 'depressive symptoms,' 'panic or anxiety symptoms,' 'medically unexplained symptoms,' or 'symptoms.' When patients did not recognize their symptoms in these definitions, they probably answered to have contacted less care providers than they actually have. Therefore, patients at risk for a depression might have contacted even more care providers than they reported. A similar recall bias can be a limitation of the study on heart failure patients.

\section{Comparison with existing literature}

As far as we know, studies describing the level of experienced continuity of care in patients at risk for depression or patients with a mental illness in primary care are lacking. The comparison of experienced continuity of care between two patient groups has also not been performed before.

\section{Interpretation of results}

The differences in experienced continuity of care that we found are small, and because of the explorative character of the study the implications of the found differences remain unclear.

The differences in personal continuity might be explained by the fact that patients at risk for depression often feel they are not listened to, and therefore, probably contact more care providers (16).

The differences in the collaboration between care providers might be explained by the fact that other types of care providers have to collaborate for patients at risk for depression (e.g. GP and psychologist/social worker) than for patients with heart failure (e.g. GP and medical specialist). Possibly, collaboration between physicians is easier because they speak the same language, whereas collaboration between a 
Uijen, A.A., Schers, H.J., Schene, A.H., Schellevis, F.G., Lucassen, P., Bosch, W.J.H.M. van depn Experienced continuity of care in patients at risk for depression in primary care. European Journal of General Practice: 2014, 20(3), 161-166

physician and a psychologist or social worker can be harder because of their different professional backgrounds.

The differences might also be explained by the fact that patients at risk for depression perceive less life satisfaction and quality of life, which might influence the answers on the continuity of care questionnaire negatively (17).

\section{Conclusion}

The explorative comparison in this study between patients at risk for depression and heart failure patients shows small differences in experienced continuity of care. This should be analysed further in a more robust study.

\section{ETHICAL APPROVAL}

Ethics committee Arnhem-Nijmegen.

Declaration of interest: The authors report no conflicts of interest. The authors alone are responsible for the content and writing of the paper.

\section{REFERENCES}

Schers H, Webster S, van den Hoogen H, Avery A, Grol R, van den Bosch W. Continuity of care in general practice: A survey of patients' views. Br J Gen Pract. 2002;52:459-62.

Uijen AA, Bosch M, van den Bosch WJ, Bor H, Wensing M, Schers HJ. Heart failure patients' experiences with continuity of care and its relation to medication adherence: A crosssectional study. BMC Fam Pract. 2012;13:86.

Uijen AA, Bischoff EW, Schellevis FG, Bor HH, van den Bosch WJ, Schers HJ. Continuity in different care modes and its relationship to quality of life: $A$ randomised controlled trial in patients with COPD. Br J Gen Pract. 2012;62:e422-e428.

Green CA, Polen MR, Janoff SL, Castleton DK, Wisdom JP, Vuckovic N, et al. Understanding how clinician-patient relationships and relational continuity of care affect recovery from serious mental illness: STARS study results. Psychiatr Rehabil J. 2008;32:9-22.

Adair CE, McDougall GM, Mitton CR, Joyce AS, Wild TC, Gordon A, et al. Continuity of care and health outcomes among persons with severe mental illness. Psychiatr Serv. 2005;56: 1061-9.

Mitton CR, Adair CE, McDougall GM, Marcoux G. Continuity of care and health care costs among persons with severe mental illness. Psychiatr Serv. 2005;56:1070-6.

Wittkampf K, van Ravesteijn H, Baas K, van de Hoogen H, Schene A, Bindels P, et al. The accuracy of patient health questionnaire-9 in detecting depression and measuring depression severity in high-risk groups in primary care. Gen Hosp Psychiatry. 2009;31:451-9.

Baas KD, Wittkampf KA, van Weert HC, Lucassen $\mathrm{P}$, Huyser J, van den Hoogen $\mathrm{H}$, et al. Screening for depression in high- risk groups: prospective cohort study in general practice. Br J Psychiatry. 2009;194:399-403.

Dowrick CF, Bellon JA, Gomez MJ. GP frequent attendance in Liverpool and Granada: the impact of depressive symptoms. Br J Gen Pract. 2000;50:361-5.

Gill D, Sharpe M. Frequent consulters in general practice: A systematic review of studies of prevalence, associations and outcome. J Psychosom Res. 1999;47:115-30.

Katon W, Von KM, Lin E, Lipscomb P, Russo J, Wagner E, et al. Distressed high utilizers of medical care. DSM-III-R diagnoses and treatment needs. Gen Hosp Psychiatry. 1990;12:355-62.

Schers HJ. Continuity of care in general practice. Exploring the balance between personal and informational continuity. Nijmegen: Radboud University Nijmegen; 2004.

Haggerty JL, Reid RJ, Freeman GK, Starfield BH, Adair CE, McKendry R. Continuity of care: A multidisciplinary review. Br Med J. 2003;327:1219-21. 
Uijen, A.A., Schers, H.J., Schene, A.H., Schellevis, F.G., Lucassen, P., Bosch, W.J.H.M. van depn Experienced continuity of care in patients at risk for depression in primary care. European Journal of General Practice: 2014, 20(3), 161-166

Reid R, Haggerty J, McKendry R. Defusing the confusion: Concepts and measures of continuity of health care, 2002. University of Columbia, Prepared for the Canadian Health Services Research Foundation, the Canadian Institute for health information and the advisory committee on health services of the federal/provincial/territorial Deputy Ministers of Health. Available at http://www.cfhifcass.ca/Migrated/PDF/ResearchReports/CommissionedResearch/cr_contcare_e.pdf (accessed 28 May 2013).

Shrive FM, Stuart $\mathrm{H}$, Quan $\mathrm{H}$, Ghali WA. Dealing with missing data in a multi-question depression scale: A comparison of imputation methods. BMC Med Res Methodol. 2006;6:57.

Johnston O, Kumar S, Kendall K, Peveler R, Gabbay J, Kendrick T. Qualitative study of depression management in primary care: GP and patient goals, and the value of listening. Br J Gen Pract. 2007;57:872-9.

Saharinen T, Hintikka J, Kylma J, Koivumaa-Honkanen H, Honkalampi K, Lehto SM, et al. Population-based comparison of health-related quality of life between healthy subjects and those with specific psychiatric or somatic diseases. Perspect Psychiatr Care. 2011;47:6673.

\section{TABLES}

Table 1. Characteristics of the study populations.

\begin{tabular}{lcc}
\hline & $\begin{array}{c}\text { Patients at risk for } \\
\text { depression } \\
(n=264)\end{array}$ & $\begin{array}{c}\text { Heart failure } \\
\text { patients } \\
(n=327)\end{array}$ \\
\hline Age, mean (SD) & $46.1(11.9)$ & $74.0(10.1)$ \\
$\leq 30$ & $31(11.7 \%)$ & $0(0 \%)$ \\
$31-40$ & $56(21.2 \%)$ & $2(0.6 \%)$ \\
$41-50$ & $75(28.4 \%)$ & $6(1.8 \%)$ \\
$51-60$ & $71(26.9 \%)$ & $25(7.6 \%)$ \\
$61-70$ & $30(11.4 \%)$ & $63(19.3 \%)$ \\
$71-80$ & $1(<0.1 \%)$ & $139(42.5 \%)$ \\
$>80$ & $0(0 \%)$ & $92(28.1 \%)$ \\
Sex & & $164(50.2 \%)$ \\
Male & $59(22.3 \%)$ & $163(49.8 \%)$ \\
Female & $205(77.7 \%)$ & \\
\hline
\end{tabular}

SD, standard deviation. 
Table 2. Pesults of items measuring personal, team and cross-boundary continuity of care. The questions on team and cross-boundary continuity were not answered by all patients, because they were not applicable to all of them (e.g. when patients did not contact a care provider in or outside general practice). We cannot distinguish between answers that are missing or not applicable, because we did no ask patients.

\begin{tabular}{|c|c|c|c|c|c|c|c|c|}
\hline \multirow[b]{2}{*}{ Personal continuity } & \multicolumn{4}{|c|}{ Patients at risk for depression } & \multicolumn{4}{|c|}{ Heart failure patients } \\
\hline & $n$ & 0 & 1 & 2 or more & $n$ & 0 & 1 & 2 or more \\
\hline 1. Total number of care providers in general practice contacted in past year for illness & 262 & $21 \%$ & $45 \%$ & $34 \%$ & 303 & $53 \%$ & $25 \%$ & $22 \%$ \\
\hline $\begin{array}{l}\text { 2. Total number of care providers contacted in pas yea for illness (in and outside } \\
\text { general practice) }\end{array}$ & 262 & $13 \%$ & $33 \%$ & $54 \%$ & 0 & Missing & Missing & Missing \\
\hline Team con tinuity in general practice & $n$ & Never/seldom & Sometimes & Often/ahways & $n$ & Never/seldom & Sometimes & Often/always \\
\hline 1. The treatment of my illness in general practice goes smoothly & 183 & $8 \%$ & $19 \%$ & $74 \%$ & 249 & $14 \%$ & $8 \%$ & $79 \%$ \\
\hline $\begin{array}{l}\text { 2. The care of the different care providers in general practice for my illnes s is } \\
\text { connected }\end{array}$ & 168 & $6 \%$ & $23 \%$ & $71 \%$ & 255 & $13 \%$ & $7 \%$ & $80 \%$ \\
\hline $\begin{array}{l}\text { 3. The care providers in general practice often give me contradictory advice about my } \\
\text { illness }\end{array}$ & 177 & $85 \%$ & $11 \%$ & $4 \%$ & 259 & $80 \%$ & $11 \%$ & $9 \%$ \\
\hline $\begin{array}{l}\text { 4. The care providers in general practice imvolved in the care for my illness } \\
\text { communicate well with each other }\end{array}$ & 165 & $7 \%$ & $21 \%$ & $72 \%$ & 257 & $11 \%$ & $9 \%$ & $80 \%$ \\
\hline $\begin{array}{l}\text { 5. The care providers in general practice involved in the care for my illness have } \\
\text { knowledge of my medical record }\end{array}$ & 173 & $10 \%$ & $17 \%$ & $73 \%$ & 264 & $9 \%$ & $12 \%$ & $79 \%$ \\
\hline $\begin{array}{l}\text { 6. The care providers in general practice involved in the care for my illness have } \\
\text { knawledge of previous visits }\end{array}$ & 169 & $10 \%$ & $18 \%$ & $72 \%$ & 253 & $13 \%$ & $11 \%$ & $77 \%$ \\
\hline Cross-boundary continuity & $n$ & Never/seldom & Sometimes & Often/ahways & $n$ & Never/seldom & Sometimes & Often/ahways \\
\hline 1. The care of the GP and the care provider outside general practice is connected & 111 & $39 \%$ & $23 \%$ & $38 \%$ & 191 & $11 \%$ & $6 \%$ & $83 \%$ \\
\hline $\begin{array}{l}\text { 2. The GP and the care provider outside general practice often give me contradictory } \\
\text { advice about my illness }\end{array}$ & 114 & $85 \%$ & $13 \%$ & $2 \%$ & 195 & $81 \%$ & $11 \%$ & $8 \%$ \\
\hline $\begin{array}{l}\text { 3. The GP and the care provider outside general practice transfer information about } \\
\text { my illness well between each other }\end{array}$ & 111 & $37 \%$ & $23 \%$ & $41 \%$ & 195 & $10 \%$ & $7 \%$ & $83 \%$ \\
\hline 4. The GP and the care provider outside general practice communicate well & 112 & $46 \%$ & $19 \%$ & $35 \%$ & 190 & $13 \%$ & $9 \%$ & $78 \%$ \\
\hline
\end{tabular}

4. The GP and the care provider outside general practice communicate well

GP, general practitioner 
Uijen, A.A., Schers, H.J., Schene, A.H., Schellevis, F.G., Lucassen, P., Bosch, W.J.H.M. van depn Experienced continuity of care in patients at risk for depression in primary care. European Journal of General Practice: 2014, 20(3), 161-166

Table 3. Mean score of team and cross-boundary continuity. The score of team continuity varied between 6 (minimum) and 30 (maximum). The score of cross-boundary continuity varied between 4 (minimum) and 20 (maximum).

\begin{tabular}{llll}
\hline & Patients at risk for depression & Heart failure patients & Difference \\
\hline Mean score of team continuity $(95 \% \mathrm{Cl})$ & $25.7(24.5$ to 27.0$)(n=172)$ & $23.7(22.8$ to 24.7$)(n=261)$ & $2.0(0.2$ to 3.9$)(P=0.03)$ \\
Mean score of cross-boundary continuity $(95 \% \mathrm{Cl})$ & $14.0(13.0$ to 15.2$)(n=110)$ & $16.1(15.4$ to 16.9$)(n=195)$ & $-2.1(-3.6$ to -0.5$)(P=0.01)$
\end{tabular}

$\mathrm{Cl}$, confidence interval

aEstimated marginal mean controlled for age and sex. 